\title{
Genotype and expression analysis of two inbred mouse strains and two derived congenic strains suggest that most gene expression is trans regulated and sensitive to genetic background
}

\author{
Harry A Noyes ${ }^{1}$, Morris Agaba22, Susan Anderson4, Alan L Archibald44, Andy Brass5, John Gibson2,6, Laurence Hall4, \\ Helen Hulme5, Sung Jong $\mathrm{Oh}^{3}$ and Stephen Kemp*1,2
}

\begin{abstract}
Background: Differences in gene expression may be caused by nearby DNA polymorphisms (cis regulation) or by interactions of gene control regions with polymorphic transcription factors (trans regulation). Trans acting loci are much harder to detect than cis acting loci and their effects are much more sensitive to genetic background.

Results: To quantify cis and trans regulation we correlated haplotype data with gene expression in two inbred mouse strains and two derived congenic lines. Upstream haplotype differences between the parental strains suggested that 30-43\% of differentially expressed genes were differentially expressed because of cis haplotype differences. These cis regulated genes displayed consistent and relatively tissue-independent differential expression. We independently estimated from the congenic mice that $71-85 \%$ of genes were trans regulated. Cis regulated genes were associated with low $p$ values $(p<0.005)$ for differential expression, whereas trans regulated genes were associated with values $0.005<p<0.05$. The genes differentially expressed between congenics and controls were not a subset of those that were differentially expressed between the founder lines, showing that these were dependent on genetic background. For example, the cholesterol synthesis pathway was strongly differentially expressed in the congenic mice by indirect trans regulation but this was not observable in the parental mice.

Conclusions: The evidence that most gene regulation is trans and strongly influenced by genetic background, suggests that pathways that are modified by an allelic variant, may only exhibit differential expression in the specific genetic backgrounds in which they were identified. This has significant implications for the interpretation of any QTL mapping study.
\end{abstract}

\section{Background}

There is considerable interest in discovering polymorphic loci that regulate differences in gene expression since this information can help us understand not only how genes are regulated but also how they interact. Genetic polymorphisms may regulate genes that are physically close to them on the chromosome (cis regulation) or anywhere else in the genome (trans regulation). There is a trivial sense in which all genes are expected to be both trans regulated by multiple transcription factors and cis regulated

\footnotetext{
* Correspondence: S.J.Kemp@liverpool.ac.uk

1 School of Biological Sciences, University of Liverpool, Liverpool, UK Full list of author information is available at the end of the article
}

by transcription factor binding sites and this has been confirmed experimentally in yeast [1]. However the distinction between cis and trans regulation is only useful when the gene is differentially expressed between two conditions; then we can ask whether that difference is due to differences in the transcription factor binding region (cis regulated) or due to differences in structure or abundance of transcription factors (trans regulated) or both.

One strategy for the discovery of cis and trans acting genes is to use gene expression as a phenotype and to map associations between thousands of markers and the expression of thousands of genes in hundreds of samples to discover expression quantative trait loci (eQTL) $[2,3]$. 
This strategy routinely detects hundreds of cis regulatory loci since it is only necessary to test a few markers around each gene, but its statistical power to detect trans regulatory loci is limited by the large multiple testing correction that is required when correlating the genotype of thousands of genome wide markers with the expression of thousands of genes. The proportions of trans regulated genes discovered in these studies vary widely depending on the significance threshold used. Furthermore it has been argued that polymorphisms in transcription factors that cause extensive phenotypic effects are likely to be rare and rapidly purged from populations [4,5]. Consequently, although polymorphic network hubs that appear to regulate large numbers of genes have been detected, their discovery is very sensitive to the analysis strategy and probability thresholds used, they have rarely been confirmed experimentally and many may be false positives $[4,6]$. Therefore most trans regulators that effect multiple genes might have small individual effects making them hard to detect by genetic mapping and give the misleading impression that cis regulation is the dominant form. These problems make eQTL studies an unsatisfactory platform for discovering the relative contributions of cis and trans regulation to the observed differences in gene expression.

The availability of data for 8 million single nucleotide polymorphisms (SNP) in inbred mouse strains has been used to construct a comprehensive haplotype map of the mouse genome [7]. This makes it possible to determine the extent to which differences in gene expression are associated with haplotype differences. However since only 15 strains were genotyped there is insufficient statistical power to approach this question directly. Instead we have divided the list of ratios of gene expression between two mouse strains into one hundred groups with increasing $\mathrm{p}$ values for differential expression and then for each group asked whether there is a significant excess of genes with haplotype differences. This strategy makes it possible to identify the $\mathrm{p}$ values associated with differences in expression and haplotype and hence the contribution of cis effects to differential expression. Importantly this strategy also makes it possible to objectively identify a threshold $\mathrm{p}$ value at which there is a significant association between haplotype differences and gene expression. This value can then be used to quantify the contribution of cis regulation to differential regulation.

We estimated the contribution of trans regulation independently using expression data from a panel of congenic mice and their controls that had been developed for other purposes. Since congenic mice have a small region of donor genome introgressed into the host genome, any differential expression that is outside the introgressed congenic region can be assumed to be trans regulated by that region, permitting a direct estimate of the proportion of trans regulated genes. These trans regulated genes were highly enriched for cholesterol synthesis genes. This suggested that the congenic region was altering cholesterol flux and this in turn was causing changes in cholesterol gene transcription a process we call indirect trans regulation. Indirect trans regulation is likely to be even more sensitive to environmental and genetic effects than direct trans regulation by a transcription factor. It is therefore important to be aware of the extent of this effect.

\section{Results}

Gene expression data were obtained for the parental and congenic lines for a total of 12 conditions (Table 1). Genes were defined as differentially expressed between lines if they had an absolute $\log _{2}$ difference in expression $>0.5$ and a pplr less than the value indicated in the text ( $p p l r$ is a measurement of probability that has the same characteristics as a $\mathrm{p}$ value, see materials and methods). The expression ratio and pplr for all relevant comparisons, together with the number of upstream SNP and the assigned haplotype is shown in Additional File 1 Expression+SNP+Haplotype.

Table 1: Microarray hybridisation conditions

\begin{tabular}{|c|c|c|}
\hline Comparison & Tissues & No. arrays \\
\hline $\mathrm{C} 57 \mathrm{BL} / 6 \mathrm{v} \mathrm{A} / \mathrm{J}$ & Liver, Kidney, Spleen & $5 / 5,5 / 5,5 / 5$ \\
\hline Tir1CC v Tir1AA & Liver, Spleen & $4 / 4,4 / 4$ \\
\hline \multicolumn{3}{|c|}{$\begin{array}{l}\text { Conditions for which expression data was obtained. Expression was compared between three pairs of strains: C57BL/6 v AJ (parental strains); } \\
\text { Tir1CC v Tir1AA and Tir3CC v Tir3AA. Pairs of values separated by a slash are the number of arrays hybridised for each of the pair of lines being } \\
\text { tested. Values separated by commas are for the different tissues. The TirnCC v TirnAA pairs were lines derived from the homozygous littermates } \\
\text { of an intercross between N7 heterozygotes for the congenic region (TirnCA animals) that acted as controls for each other. Hence Tir1CC and } \\
\text { Tir1AA mice are carriers of C57BL/6 and A/J alleles respectively at the Tir } 1 \text { locus. }\end{array}$} \\
\hline
\end{tabular}




\section{Effect of sequence polymorphism on the probability of differential expression between inbred lines}

Any SNP that causes a mismatch between probe and target might have a direct effect on the measure of expression by reducing signal and hence confound the observations [19], however Affymetrix arrays are probably relatively insensitive to this effect [6]. Since probes were designed against the C57BL/6 sequence any effect of mismatches on signal would be expected to lead to a loss of signal from $\mathrm{A} / \mathrm{J}$ and an excess of genes that appear to be over-expressed in C57BL/6. The positions of each Affymetrix 25 mer probe in probesets that were scored as differentially expressed between A/J and C57BL/6 liver were retrieved from the Ensembl39 (NCBI36) database and compared with the position of SNP in the Perlegen (NCBI36) database. 59 out of the 797 differentially genes had SNP under probes but there was no evidence for an excess of SNP associated with probesets that were underexpressed in $\mathrm{A} / \mathrm{J}\left(\mathrm{x}^{2} 0.21,1 \mathrm{df}, \mathrm{p}=0.64\right)$. Furthermore $49 \%$ more genes were over-expressed in $\mathrm{A} / \mathrm{J}$ than $\mathrm{C} 57 \mathrm{BL} / 6$; the opposite direction to any effect that would be expected to be caused by SNP modifying expression. Consequently the data could be used with confidence that observations were not a simple consequence of SNP causing expression differences (Additional File 2 SNP_under_Probes).

\section{Association between upstream SNP and differential expression}

Our objective was to identify any association between the number of upstream SNP and the probability of genes being differentially expressed. The genes represented in the liver data were ranked by pplr and divided into 100 groups of 193 genes each, representing the percentiles of the $p p l r$ distribution. For each percentile group the number of genes with each number of SNP between 1 and 10 in the $1 \mathrm{~kb}$ upstream region was obtained. For each percentile the number of genes with at least 1 upstream SNP was compared with the number with no upstream SNP by a $X^{2}$ test. Then this was repeated for each number of upstream SNP between 2 and 10 Additional File 3 SNP_and_Expression). After a Bonferroni correction for multiple testing, there was only a significant association between upstream SNP and differential expression for the first three (most significant) percentiles of the pplr distribution, containing 580 genes with pplr $<0.0046$, of which 246 were on different haplotypes. The relative risk of a gene being differentially expressed was obtained for genes with each number of SNP (Fig. 1). There was a strong correlation between the relative risk and the number of SNP in the upstream region for the first percentile $(r=0.99)$ and a similar relationship was observed for the next two but not subsequent percentiles.

The $\chi^{2}$ tests showed highly significant associations with the presence of SNP and differential expression when

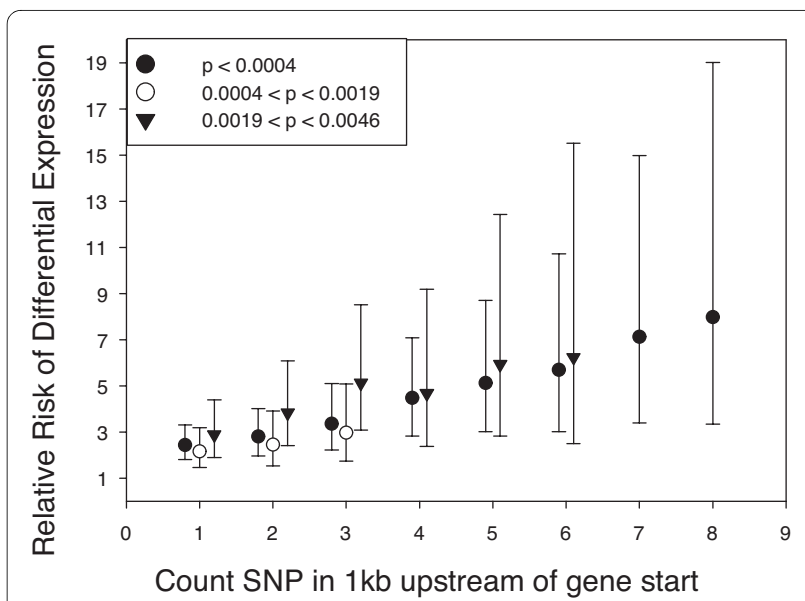

Figure 1 Relative risk of differential expression for genes with $p p l r<0.0046$. The relative risk that a gene would be differentially expressed given that it had $n$ or more informative SNP in its $1 \mathrm{~kb}$ upstream region is shown for genes within the first three percentiles of the pplr distribution ( $p p / r<0.0046)$. The points on the graph have been spread around the integer values to make the individual error bars visible. There was no significant effect on relative risk for genes that had a pplr $>0.0046$ after a Bonferroni correction for multiple testing. The error bars represent the $95 \%$ confidence interval of the relative risk.

$p p l r<0.005$. However there was no evidence for an association between the presence of SNP and genes that had $p p l r>0.005$. This was interpreted as evidence that the genes that are differentially expressed under the immediate control of upstream SNP have highly reproducible expression differences and hence give rise to low pplr values. In order to test this hypothesis the proportion of genes that were differentially expressed between the Tir1CC and Tir1AA mice and inside or outside the Tir1 congenic interval were compared for genes with pplr < 0.005 and $0.005<p p l r<0.05$. Of the 112 genes that were differentially expressed in Tir 1 spleen or liver at the pplr< 0.005 confidence level, 23 were in the Tir 1 congenic interval whereas of the 328 that were differentially expressed at the $0.005<p p l r<0.05$ confidence level only 7 were in the congenic interval (Fisher Exact Test; $\mathrm{p}<10^{-9}$ ). This provided persuasive evidence that low pplr values are associated with haplotype differences.

\section{Estimating the frequency of differentially cis regulated genes between parental inbred mice}

The haplotype of the $1 \mathrm{~kb}$ upstream region differed between C57BL/ 6 and $\mathrm{A} / \mathrm{J}$ for $30 \%$ of all genes for which haplotype data were available. This value is likely to be an estimate of the proportion of genes regulating any phenotype that can be discovered in a cross between these two strains. The extent to which genes are cis regulated may be reflected by the excess of those genes that are both differentially expressed and on different haplotypes over the number that would be expected to be on different haplo- 
types by chance. In the parental mice $30 \%$ of all genes were on different haplotypes in the two strains, but 55\% of genes that were differentially expressed in at least one tissue were also on different haplotypes $\left(x^{2} 417,1 \mathrm{df} p<\right.$ $\left.10^{-93}\right)$. Applying Bayes theorem shows that approximately $36 \%$ of genes $(30 \%, 36 \%$ and $43 \%$ for spleen, kidney and liver respectively) that are differentially expressed in any one tissue can be attributed to haplotype difference and hence putatively cis-regulated (Fig. 2 and Additional File 4. Expression_and_Haplotype). $83 \%$ of genes that were differentially expressed in all three tissues tested in the parental strains had different haplotypes for the $1 \mathrm{~kb}$ upstream region and $61 \%$ of these genes may be differentially expressed due to the haplotype differences (Fig. 2). $51 \%$ of genes that were differentially expressed in two tissues were predicted to be due to difference in haplotype.

Genes that were potentially cis regulated, i.e. those with differential expression and on different haplotypes, were also five times more likely to be differentially expressed in

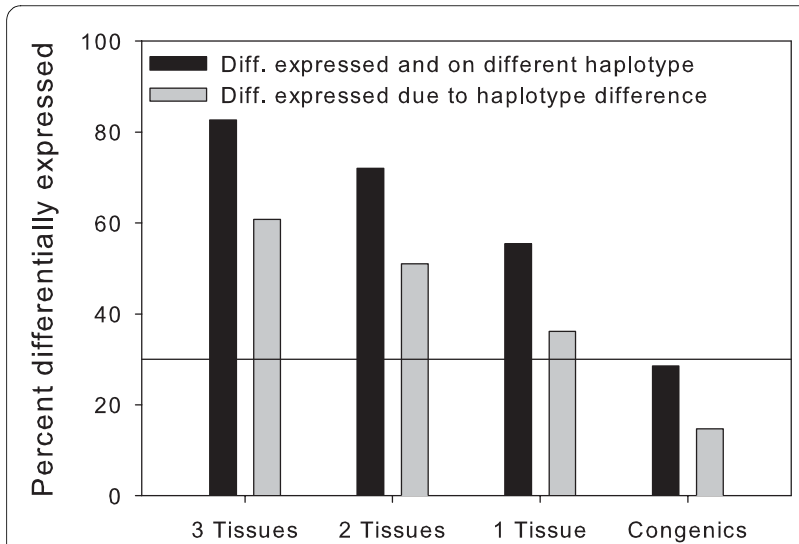

Figure 2 Frequency of differentially expressed genes on different haplotypes and attributable to haplotype difference. The proportion of the genes that were differentially expressed and also on different haplotypes in the parental C57BL/6 and A/J mice was obtained for all combinations of the three tissues at pplrthresholds of 0.005 (see Fig $3 a$ for numbers). The percentages of genes that were differentially expressed and on different haplotypes are shown by the solid black bars, the percentages of genes that were estimated using Bayes Theorem to be differentially expressed due to haplotype differences are shown by the grey bars. $30 \%$ of the genes in $\mathrm{A} / \mathrm{J}$ mice were on different haplotypes from C57BL/6, indicated by the horizontal bar on the plot. There was a significant excess of differentially expressed genes on different haplotypes in the parental mice but not in the congenic mice when the congenic region was excluded. Key "3 tissues" (kidney, liver, spleen) in parental mice (C57BL/6 and A/J); "2 tissues" - mean of the three combinations of two out of three tissues from the parental Mice; "1 tissue" - mean of individual tissues from the parental mice; "Congenics" mean of the genes that were differentially expressed in the congenic mice and on different haplotypes in the parents for for the Tirl (liver and spleen) and for Tir3 (liver) congenic excluding the congenic region. The percentage of genes that are differentially expressed in the congenic mice and on different haplotypes in the parents is expected to approximate to the percentage of genes on different haplotypes in the parents as was found to be the case. multiple tissues than genes that are potentially trans regulated: $46 / 268$ (17\%) of genes that were differentially expressed in at least one tissue and on different haplotypes (putatively cis regulated) were differentially expressed in all three tissues, whereas only $7 / 214$ (3\%) of genes that were differentially expressed in at least one tissue and on the same haplotype (putatively trans regulated), were differentially expressed in all three tissues (Fisher exact test, $\mathrm{p}=10^{-7}$ ). This suggests that cis effects were five times less sensitive to tissue environment than trans regulated genes.

\section{Effect of genetic background on differential expression}

The number of genes that were differentially expressed in each condition or in relevant combinations of conditions are summarised in figure 3. As expected, there was little overlap in the lists of genes differentially expressed in different tissues. In the parental lines for example (Fig. 3a), $85 \%$ of all differentially expressed genes are differentially expressed in only one of the three tissues.

Surprisingly however, there was relatively little overlap between the sets of genes that were differentially expressed in the congenic strains and in the parental strains from which they were derived (Fig. 4). In the spleen only $3 / 77$ genes that were differentially expressed in the congenic mice were also differentially expressed in

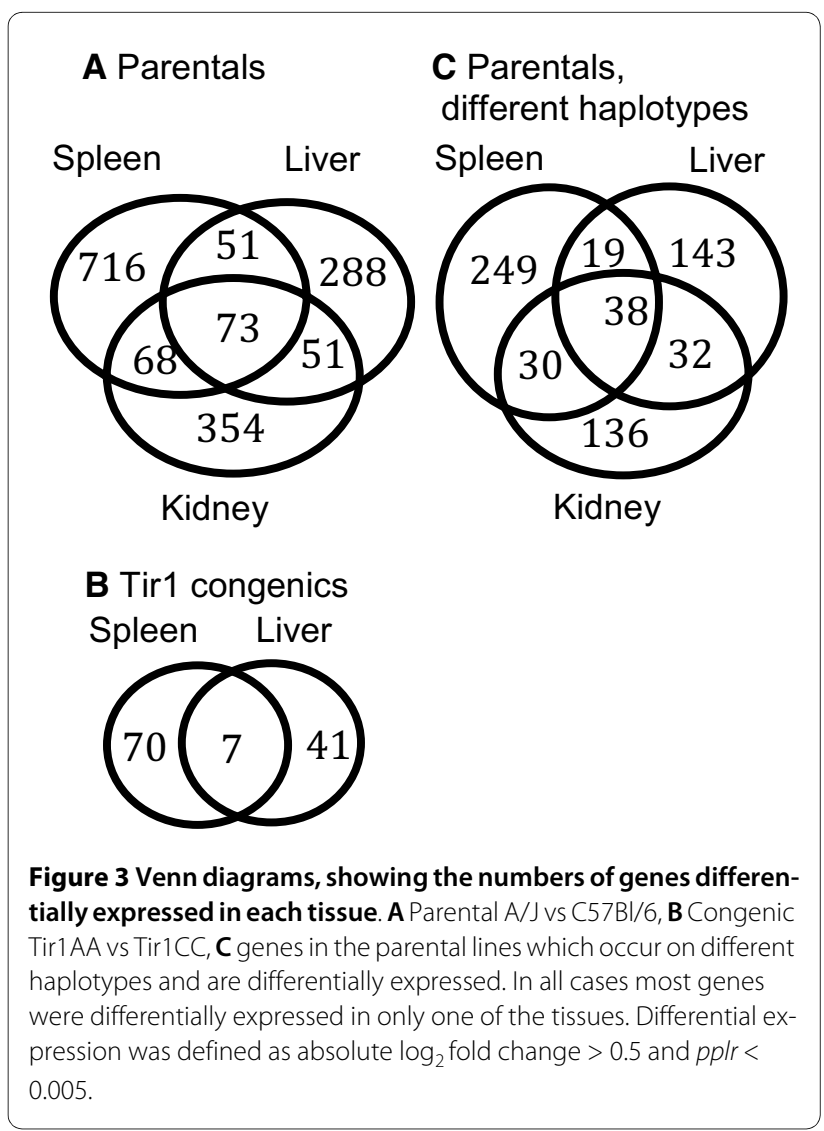




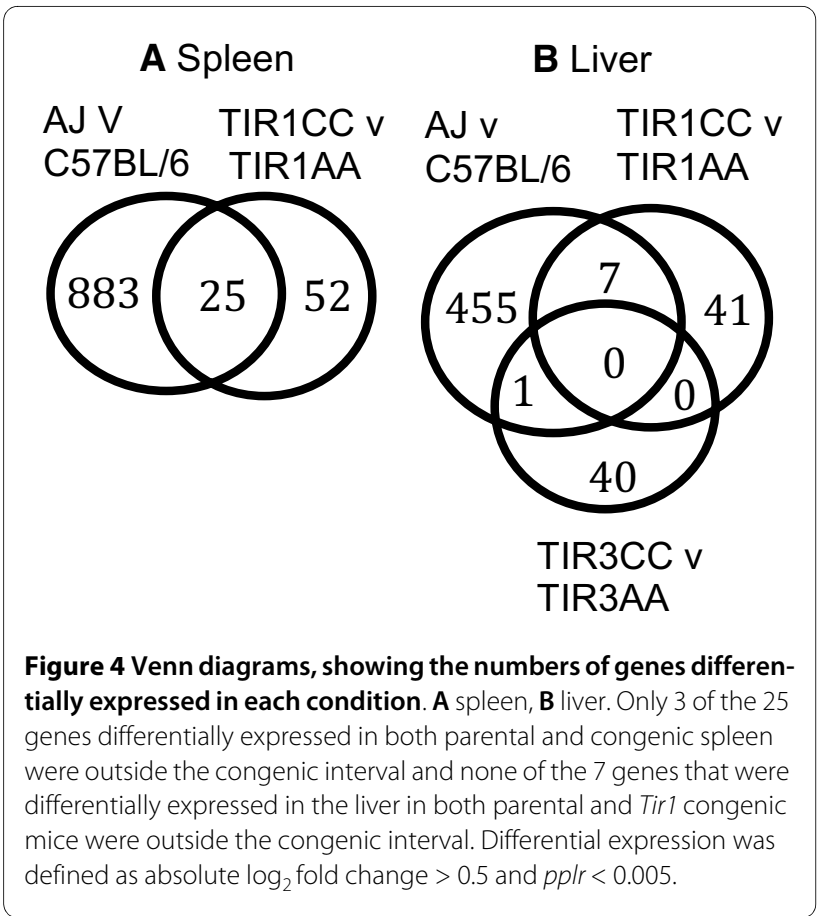

the parents and outside the congenic interval. In the liver there were no genes that were outside the congenic interval and differentially expressed in both congenics and parents. This is less than would be expected by chance in each case and shows that the trans effect of genes within the congenic interval on genes outside the interval is not detectable in the parental mice. Microarray studies to detect pathways that cause the difference in phenotype between strains are based on the assumption that the difference in expression of those pathways will be substantial enough to be observable. In this case there was no observable difference in the parental strains in any of the pathways that were regulated by the QTL in the congenics and therefore expression data from the parental strains may not be a reliable resource for identifying the pathways that regulate the phenotype.

\section{Estimating the proportion of trans regulated genes}

In the spleen of the congenic mice 55 of 77 (71\%) differentially expressed genes were outside the congenic interval and therefore putatively trans regulated. In the congenic liver (Fig. 4b) 41/48 (85\%) of genes appeared to be trans regulated. The most prominent group of trans regulated genes was the cholesterol and steroid synthesis pathway, which was significantly differentially regulated $\left(\mathrm{p}=2.55^{-7}\right)$ in the liver between Tir1CC and Tir1AA mice but no difference in this pathway was observed between the parental or the Tir3NN strains (Additional File 5 GO_KEGG). Eleven of the thirteen genes in the steroid biosynthesis pathway between $H m g c s 1$ and Dhcr7 were significantly over-expressed in the liver of Tir1AA mice

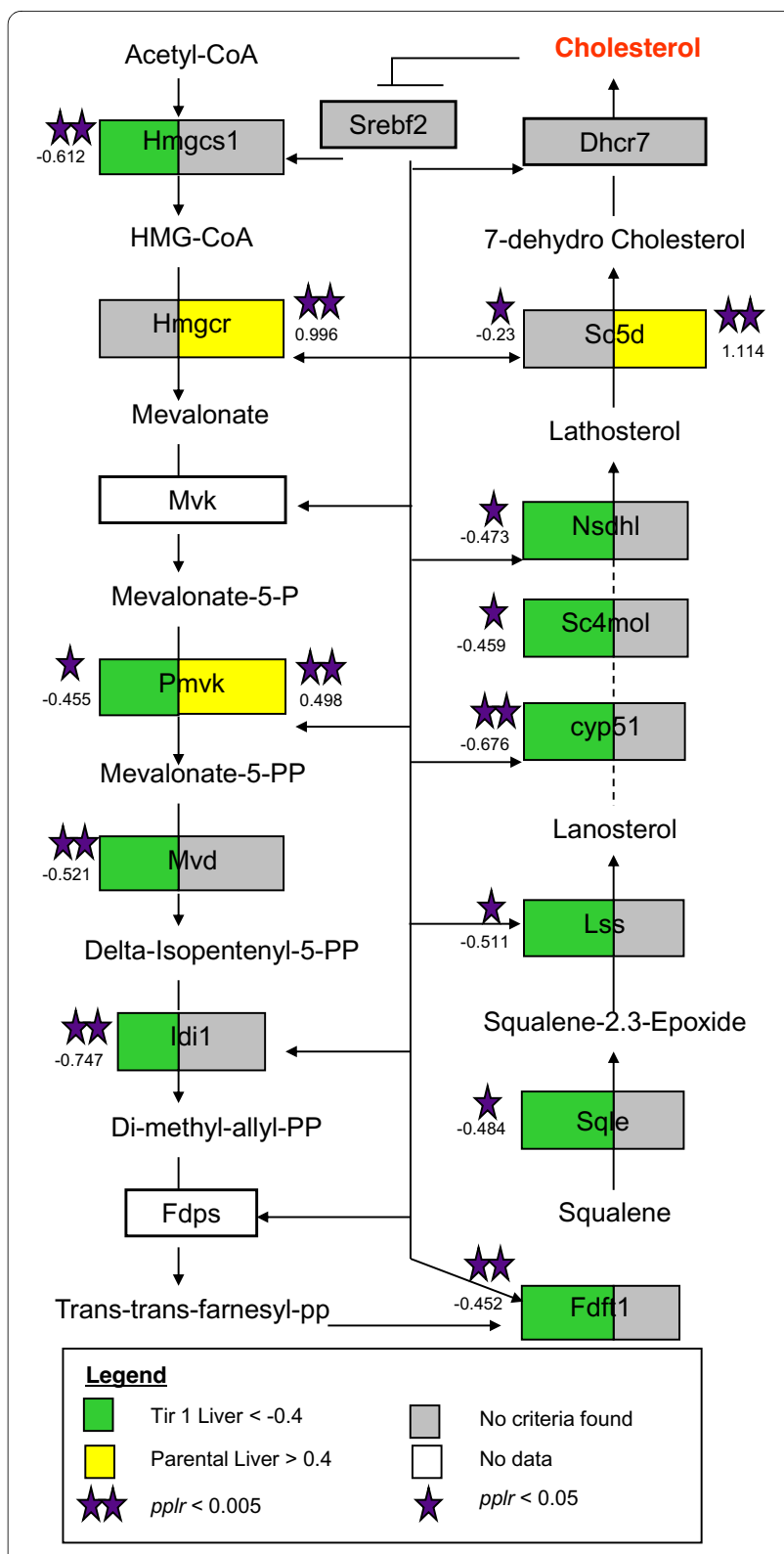

Figure $\mathbf{5}$ Cholesterol synthesis pathway compiled using GenMapp. Genes regulated by Srebf2 (Srebp2) from Reed et al. [33]. Ratio of Tir1CC to Tir1AA congenic mouse expression is shown on the left of each box and the ratio of C57BL/ 6 to $\mathrm{A} / \mathrm{J}$ parental mouse expression is shown on the right of each box. Genes in green boxes have $<-0.4 \log _{2}$ expression ratio in the liver of the congenic mice, genes in yellow have $>0.4 \log _{2}$ expression ratio in the liver of parental mice. Genes indicated by purple stars are significantly differentially expressed as indicated in the legend. $\log _{2}$ expression ratios are shown by each gene where the absolute ratio $>0.4$ or the ratio is significantly different from 0 as indicated by the stars. Negative $\log _{2}$ ratios indicate higher expression in mice carrying $\mathrm{A} / \mathrm{J}$ alleles (A/J and Tir1 AA) than mice carrying $\mathrm{C} 57 \mathrm{BL} / 6$ alleles (C57BL/6 and Tir1CC). None of the genes in this pathway were differentially expressed in Tir3AA $\vee$ Tir3CC.

$p p l r<0.05$ (Fig. 5). In contrast only 3 out of the thirteen had significantly higher expression in parental C57BL/6 
than in $\mathrm{A} / \mathrm{J}$, the opposite direction of effect and not enough differentially expressed genes to cause the pathway to be flagged as differentially expressed.

\section{Discussion}

The availability of high-density genotype data for mice has made it possible to quantify the relationship between haplotype structure and differential gene expression. A relationship between SNP in the $1 \mathrm{~kb}$ upstream region and differential expression was only observable when using a stringent test of differential expression (absolute $\log _{2}$ fold change $>0.5 ; \mathrm{pplr}<0.005$ ). This was interpreted as evidence that the variance of expression of cis regulated genes is much lower than that of trans regulated genes. Although the $1 \mathrm{~kb}$ upstream region may contain the highest density of regulatory elements it does not contain all of them, they can be spread throughout the gene and its 3' region as well as going tens to hundreds of kilobases upstream. Therefore we are likely to have underestimated the numbers of cis regulated genes using this strategy. However SNP in the upstream region will frequently be markers for larger haplotypes that extend into or through the whole gene region so these regions will not have been completely excluded from the analysis. By selecting the region with the highest density of regulatory elements we have maximised our power to detect an association between SNP and gene expression, which would have been more difficult in the presence of higher proportions of non-functional SNP distributed through the rest of the gene region.

If SNP within probe positions were directly affecting signal then this would confound our results. Since the probes were designed against C57BL/6 sequence any SNP that effected signal would be expected to reduce signal in $\mathrm{A} / \mathrm{J}$ relative to $\mathrm{C} 57 \mathrm{BL} / 6$. However we have shown that only 59 of the 797 differentially expressed genes in the liver had SNP under probes and even for these 59 there was no association between their presence and reduced signal in $A / J$. Therefore we do not consider that SNP under probes is a source of bias in our data.

By examining the correlation between gene expression differences and haplotype allele differences in $A / J$ and C57B/6 mice we estimated that haplotype differences accounted for $36 \%$ of expression differences and these were assumed to be cis regulated, although as noted above this is likely to be an underestimate. We independently estimated that 71 and $85 \%$ of genes were outside the congenic interval and hence trans regulated in the spleen and liver of the Tirl congenic mice respectively. This also likely to be an underestimate since some genes within the congenic interval may also be trans regulated. The combined data suggests that there might be an approximately 2:1 ratio of trans:cis regulated differences in gene expression. Clearly this is a preliminary estimate based on a very small sample but it emphasises the dominance of trans regulation.

Genes are both cis and trans regulated and by multiple cis binding sites and multiple trans acting factors. However the 2:1 ratio may reflect the contributions of trans and cis factors to the observed differences in expression. It is well established that there are large differences in gene expression between tissues [20] and this observation was replicated here. Indeed this must be so for cell types to differentiate, thus underlining the sensitivity of gene expression to environment and this regulation must be by a trans mechanism. A study of mouse brain regions in multiple mouse strains found that region specific transcription was mainly trans regulated whilst strain specificity was mainly cis regulated [20].

The cholesterol synthesis pathway appeared to be trans regulated by the Tir1 region in the congenic mice but this was not observable in the parental mice. This pathway is regulated by the transcription factor SREBF2, which is inactive when cholesterol concentrations are high but migrates to the nucleus when cholesterol is low to bind transcription factor binding sites in cholesterol pathway genes and promote transcription; thus forming a negative feedback loop on cholesterol synthesis [21]. The co-ordinated up-regulation of the cholesterol pathway in Tir1AA mice implies that SREBF2 is responding to lower cholesterol levels in the livers of Tir1AA mice than Tir1CC mice. Since cholesterol synthesis is co-ordinately regulated by an end-product feedback mechanism through SREBF2, the most likely explanation for the co-ordinated response is that some gene or genes in the Tir1 congenic region is modifying cholesterol flux (but not necessarily serum cholesterol levels) by changing the rate of absorption or excretion from cells or the body. There are at least two genes in the Tirl region that are directly involved in cholesterol metabolism (Abcg1 which participates in cholesterol efflux from the cell and $R x r b$ which regulates $A b c$ mediated efflux[22]) but cholesterol plays an important role in the response to infection and Tnfa which is also in the Tir1 region has been shown to regulate cholesterol as well $[23,24]$, any of the other immune related genes in the MHC region could also be contributing to changes in cholesterol flux. We cannot exclude the possibility that a novel transcription factor in the Tir1 region may be competing with SREBF1 to directly regulate cholesterol pathway transcription. However, since there is no evidence in our data to suggest that a novel mechanism is responsible for our observations, it is most parsimonious to assume that the standard model holds here and the cholesterol pathway is responding to changes in cholesterol flux through the liver. Since no significant difference in cholesterol pathway expression was observed in the parental strains we assume that multiple other interacting processes are buffering the effect of the Tir1 region in these 
lines, as would be expected if the region was acting by modifying cholesterol flux rather than acting through a transcription factor.

We describe this mechanism of regulation by multiple intermediates such as metabolites as indirect trans regulation to distinguish it from direct trans regulation of a target gene by a transcription factor. Cholesterol synthesis is a particularly well-known example of indirect trans regulation [25].

Almost completely different sets of genes were found to be differentially expressed between the congenic mice and their controls and between the parental inbred mice, as has been observed before [26]. This suggests that the problem of identification of trans regulated genes goes well beyond the lack of statistical power of eQTL studies for this purpose. Expression differences in cis regulated genes were found to have lower variances than those of trans regulated genes in this study, and several eQTL studies have found that cis regulated differences in transcription are larger than trans regulated differences [3]. This is to be expected since trans regulated genes are likely to have a much larger number of intermediates involved in their effect on expression. Firstly there is usually a complex of gene products and metabolites that bind to a given regulatory factor binding site, eg a mean of 3.1 loci have been found to regulate each trans regulated gene in radiation hybrid cells [27]. Secondly the concentrations and activities of members of that complex may be regulated by other molecules elsewhere that regulate translocation to the nucleus. Consequently the regulatory binding site acts as a transponder for the whole cascade of events leading to regulatory complex binding or activation, for example a study in yeast found that trans acting eQTL were not enriched for transcription factors and that a wide range of gene classes could cause trans differences in expression [1]. This suggested that most differences in gene expression were caused by indirect rather than direct (transcription factor mediated) trans effects. Each interaction in the regulatory cascade is likely to introduce an element of noise and hence the effect of a trans acting regulatory factor polymorphism on gene expression is likely to have much higher variance than a cis polymorphism. Therefore indirect trans regulation is likely to have even larger variance than direct trans regulation. These higher variances will make trans regulated differences in expression between conditions much harder to detect than cis regulated differences and indirect trans harder than direct trans. We do not know the proportion of genes that were indirectly trans regulated in the congenic mice; there are several zinc finger proteins with unknown targets in the congenic region that might be direct trans regulators. Indirect trans regulation relationships are likely to be less well known because they are more complex and harder to discover experimentally by methods such as chromatin immunoprecipitation. But if the cholesterol synthesis pathway is representative of the main mode of trans regulation in the congenic mice, most regulation was indirect.

The greater sensitivity of trans regulated expression to experimental conditions than cis regulated genes may make it even harder to detect those genes that are subject to both polymorphic trans and cis factors. Since the penetrance of the cis acting factors is usually likely to be higher than the trans factors only the strongest trans effects will be observable in the presence of concurrent cis effects.

The large proportion of trans regulated genes is important because their regulation appeared to be less stable under different conditions and consequently gene expression based approaches to the discovery of genes regulating a phenotype are likely to be exquisitely sensitive to the particular experimental conditions used. There was almost no overlap between genes that were differentially expressed between congenics and controls and outside the congenic regions, and genes that were differentially expressed between the parental inbred mice strains, even when comparing the same tissue, and this is consistent with previous observations [26]. This indicates that the observable trans regulation of gene expression is highly context dependent; the context in this case being genetic background. A study of recombinant inbred strains of mice estimated that 1500 genes differed between conditions and 1200 were sensitive to genetic background and hence presumably trans regulated [28]. The sensitivity of phenotype to genetic background in which a trait is expressed has also been dramatically illustrated by a study of 41 selected traits in a complete panel of chromosome substitution strain mice (CSS) that found that for $56 \%$ of phenotypes the sum of the trait differences from the host strain over all CSS lines exceeded $500 \%$ of the difference between the host and donor strain [29].

The sensitivity of both expression and phenotype to genetic background has profound implications for the interpretation of expression data for a range of purposes. For example, the use of expression arrays is a common strategy for following-up QTL mapping studies with the objective of identifying the genetic polymorphism underlying a QTL. Gene expression is typically measured in the parental lines that are used to generate the mapping populations in order to infer the genes regulated by the QTL. The candidate quantative trait gene(s) (QTG) causing the phenotypic difference may be identified because they are differentially expressed in at least one tissue. Candidate genes can be selected from these long lists by identifying those that participate in networks that are differentially expressed and that intersect with the QTL region. We have previously identified a candidate gene (Daxx) in the Tir1 QTL region that is not differentially expressed, but 
was subsequently found to have an amino acid indel, on the basis of its membership of a differentially expressed KEGG pathway in the parental strains [30]. However it is clear from the data presented here that there is no reason to expect that the genes and pathways that are regulated by the QTL gene(s) will respond in the same way in the parental mice as they will in the mapping populations and hence the pathways that differ between the extremes of the mapping population may not be detected by measuring gene expression in the parental mice.

The observation that the gene expression phenotype of congenic mice is overwhelmingly not predictable from the expression of genes in the parental strains has consequences for both QTL discovery and exploitation. It begs the question of why congenic mouse lines often retain the expected trait associated with the introgressed QTL. The explanation may be that most QTL that are discovered in mapping populations are those that are caused by alleles that are insensitive to genetic background. This would explain why mapping studies that have used parental strains with limited genetic differences have discovered unusually large numbers of QTL [31]. This could mean that conventional QTL mapping approaches fail to map the bulk of the theoretical potential of any given quantitative trait, not because the unmapped fraction is associated with multiple loci of small effect but because it is associated with loci that may be of large effect only on particular genetic backgrounds. If this is the case then the large number of inbred mouse strains being generated by the collaborative mouse cross [32] are likely to reveal far more loci regulating phenotypic differences than a simple comparison between the eight founder lines would predict.

\section{Conclusions}

We have found that cis regulated genes are associated with low pplr values $(<0.005)$, presumably because the close coupling of the polymorphic regulatory region to the gene leads to much lower variances in expression. There was a ratio of approximately 2:1 of trans:cis regulated genes and the cis regulated genes were more likely to be differentially expressed in multiple tissues than trans regulated genes. The fact that genes that are regulated by a congenic region were not observably regulated in the parental mice, means that expression studies in the parents of a mapping population are unlikely to detect many of the trans regulated expression differences caused by the QTL genes. This will make it much harder to identify the pathways regulated by a QTL. The Tir1 region of chromosome 17 regulated the cholesterol synthesis pathway, but this was probably because this region modifies cholesterol flux and not because it contains transcription factors that regulate cholesterol metabolism. This mode of indirect trans regulation may be the most common form of gene regulation but also the hardest to detect except in well defined genetic backgrounds such as congenic mice. Consequently only the cis and largest direct trans regulatory relationships will be amenable to discovery and the large fraction of indirect trans regulation will be missed by most high throughput experimental strategies.

\section{Methods \\ Congenic mice}

All mouse work was conducted at the International Livestock Research Institute in Nairobi and approved by their Internal Animal Care and Use Committee. In the UK no project licence would be required for the procedures described here since they were all conducted on post mortem animals that had not been subject to any prior treatment.

Two congenic lines were created to cover the Tir 1 and Tir3 QTL for Trypanosoma infection response [8,9] as previously described [10]. Two congenic lines were created corresponding to predicted Trypanosoma infection response (Tir) loci; Tir1 and Tir3 on chromosomes 17 and 1 , respectively. The progeny at each backcross generation were genotyped with microsatellite markers defining the genetic intervals containing the QTL using the following markers: D17Mit29, D17Mit16 and D17Mit11 (Tir1); D1Mit60, D1Mit217 and D1Mit87 (Tir3). At the seventh generation of backcrossing each line was typed with a series of markers at approximately $2 \mathrm{cM}$ intervals flanking the Tir loci. The individuals with the shortest donor haplotype extending beyond the QTL interval were used for breeding the next generation by intercrossing a single heterozygous male with full or half sib female carriers of the $\mathrm{C} 57 \mathrm{BL} / 6$ donor region. The progeny of these were genotyped and those individuals homozygous for the alternative haplotypes were used as founders to propagate each line, which were denoted either TirnAA or TirnCC for homozygotes at the QTL for recipient A/J haplotype and the donor $\mathrm{C} 57 \mathrm{BL} / 6$ haplotype, respectively. Thus in total 4 lines were produced; these are Tir1AA and Tir1CC; Tir3AA and Tir3CC. The Tir1CC line is homozygous for a C57BL/6 haplotype spanning 10 cM interval between marker D17Mit84 and D17Mit177 on Mmu17. Tir3CC individuals have a C57BL/6 haplotype spanning approximately $10 \mathrm{cM}$ between markers D1Mit49 and D1Mit139 on Mmu1.

The recommended names for the test lines according to the Mouse Genome Informatics would be A.B6-Tir1 and A.B6-Tir3. However there is no recommended nomenclature for the control lines, so for clarity the TirnAA and TirnCC style will be used in the following description.

The use of controls derived from the same line as the congenic mice is critical since even the small amount $(<1 \%)$ of non target $\mathrm{C} 57 \mathrm{BL} / 6$ remaining can have pro- 
found non-specific effects on phenotype through heterosis. The homozygous congenic mice lines were genotyped at the Wellcome Trust Clinical Research Facility, Edinburgh, UK using the Illumina Mouse Medium Density Linkage Panel on an Illumina BeadStation 500 instrument, with the Illumina 1536 murine SNP panel. 959 markers were informative between A/J and C57BL/6 with a mean spacing of $2.61 \mathrm{Mb}$. Using this data it was possible to identify the approximate boundaries of the introgressed regions and also to identify non-target regions of C57BL/6 origin that had also been carried through into the congenic lines.

This showed that Tir1CC congenic mice carried a region of $\mathrm{C} 57 \mathrm{BL} / 6$ between 26.0-43.9 $\mathrm{Mb}$ (NCBI36) on Mmu17 in the A/J background, this region includes the major histocompatibility complex (MHC). The Tir3CC mice carried a region of $\mathrm{C} 57 \mathrm{BL} / 6$ origin between 93.3123.6 Mb on Mmu1. The SNP genotyping data showed that approximately $0.75 \%$ of the genome outside the congenic regions was of $\mathrm{C} 57 \mathrm{BL} / 6$ origin in both TirNAA and TirNCC mice [10]. The non-target regions were not fixed in the mice that were genotyped and no differentially expressed genes were observed in these regions. It is impossible to exclude the possibility of trans effects from these loci, however since they were present but not fixed in both test and control mice we would have had very low power to detect trans effects from the non-target loci, therefore we have disregarded possible effects from these loci in the subsequent discussion.

The initial cross and the first three backcrosses were reciprocal between the sexes, the fourth backcross was of congenic females to $\mathrm{A} / \mathrm{J}$ males to eliminate residual C57BL/6 Y chromosomes. Subsequent backcrosses were of a single congenic male to multiple A/J females. The $\mathrm{Y}$ chromosome was not assayed in the SNP genotyping so the $\mathrm{Y}$ chromosome genotype has not been independently verified.

\section{Expression analysis}

Affymetrix Mouse 4302.0 microarrays were used for expression profiling of each of the two congenic lines, their respective controls and the parental A/J and C57BL/ 6 mice as part of a larger study of response of the transcriptome to infection with Trypanosoma congolense [11]. C57BL/6JOlaHSD (C57BL/6) and A/JOlaHsd (A/J) mice were purchased from Harlan UK housed at the ILRI facility on a twelve-hour light/dark cycle and fed mouse diet SDS-RM3 (E) (4.2\% fat, 22.4\% protein; Special Diets Services, Witham, Essex UK) and water ad libitum for 4 weeks to acclimatise before killing. Tissues for hybridisation were selected iteratively and different sets of tissues were hybridised for each pair of strains (Table 1). RNA from liver, spleen and kidney of the parental lines were hybridised first and the data showed that the liver, spleen and kidney varied in the differences that they exhibited between strains. The kidney differed least between parental strains and was excluded from the subsequent experiments on the congenic mice. The liver was found to give the most reproducible signals, with the most differentially expressed genes and be informative for many immune related functions and was hybridised for all lines. The differences in the liver between Tir3 test and control mice were very small and consequently spleen data was not collected for these animals.

Congenic mice were used at the second generation after being made homozygous. Congenic mice were co-housed under the same conditions as the parental mice and equal numbers of each line were simultaneously killed for tissue collection in three batches 1 year after the inbred mice at a mean age of 20 weeks. All batches of mice were killed at $2 \mathrm{pm}$ to minimise diurnal variation. For each strain RNA was prepared from various tissues of twenty or twentyfive mice. Tissues were ground under liquid nitrogen and extracted with Trizol (Invitrogen) and further purified on RNAeasy columns (Qiagen). RNA was quantified and checked for integrity on an Agilent Bioanlyser and extractions were repeated for any degraded samples. The samples were then mixed into four or five pools of five samples for each strain, most pools comprised of samples of a single sex. The pooling strategy that we used is predicted to give the same power to detect differential expression as over fifteen individual samples hybridised separately [12]. Sex was balanced in each set. For the congenic mice we compared chips by sex to identify sex effects as well as by strain to identify strain effects and also mixtures of strain and sex to identify background noise. Twice as many genes were differentially expressed between the sexes as between the strains but there was no overlap between the two sets, ie no genes that were differentially expressed between the sexes were also differentially expressed between congenic test and control lines. Consequently we are confident that our observations are not due to sex effects.

Chips were initially assessed using DChip criteria and PCA to screen for outliers. Data from outlier chips were discarded and hybridisations were repeated with the same or fresh RNA samples from the same batch of mice until complete sets of chips for each condition were obtained. Sets of 25 mer probes for each gene represented on the array were identified using AffyProbeMiner [13]. Normalisation was carried out in the $\mathrm{R}$ environment using multi-mgmos [14] and differential expression was also assessed in $\mathrm{R}$ using PPLR (Probability of Positive Log-Ratio) [15]. The PPLR method uses Bayesian techniques to detect differentially expressed genes. The method assigns a confidence statistic, taking values between 0 and 1, based on the reproducibility of the probe-level measurements. A score of close to 0 or close 
to 1 indicates evidence of an exceptional event, namely up-regulated or down-regulated expression between conditions respectively. Common events have values around 0.5 . In the analysis presented here we have used the minimum of (PPLR, 1- PPLR), which we denote pplr as a confidence value, for ease of comparison and ranking of genes such that a value close to zero indicates significant confidence in differential expression. PPLR makes use of the probe-level measurement error across replicated experiments and may be most appropriate where there are a variable number of probes per probeset, as is the case with probesets obtained from AffyProbeMiner. The data is available through ArrayExpress under accession numbers E-MEXP-1190 for the parental strains and ETABM-865 for the congenic strains.

\section{Associations of SNP and Expression}

The Perlegen SNP dataset [7] was downloaded into a MySQL database. A list of all genes in the genome together with their start positions and strand was similarly downloaded from Ensembl using the NCBI36 mouse genome assembly. For each Affymetrix probe set, the number of SNPs between C57BL/6 and A/J in the $1 \mathrm{~kb}$ upstream region of the gene to which the probe was targeted was obtained from the database. This information was used to obtain the counts of genes with absolute $\log _{2}$ fold difference between strains $>0.5$ and pplr values for a difference in expression below the thresholds indicated in the text.

\section{Associations of haplotype and expression}

Boundaries of haplotypes identified within the Perlegen SNP data set were downloaded from the UCLA Perlegen Mouse SNP Browser Mouse[16], strains were allocated to haplotypes at each haplotype block using a local Perl script that extracted all alleles from the Perlegen dataset within a haplotype block, aligned them on the basis of genomic positions provided with the data and submitted them to the Jukes-Cantor algorithm in DNADIST in PHYLIP to calculate genetic distances between strains $[17,18]$. The distribution of distances was examined and a threshold of 0.2 was selected, such that strains within a distance of 0.2 were allocated to the same haplotype block using C57BL/6 as the reference strain (See Additional File 6 Haplotype block assignment). Haplotype assignments for each Haplotype block are shown in Additional File 7 Haplotype_block_alleles, 13,385 Ensembl genes could be assigned to haplotypes.

\section{Estimating the frequency of differentially cis regulated genes using Bayes theorem}

Bayes theorem was used to estimate the proportion of differentially expressed genes that were putatively differentially expressed because of difference in haplotype.
Bayes Theorem: $\left.\left(\left(\mathrm{N}_{\mathrm{h}} / \mathrm{E}\right) \times\left(\mathrm{N}_{\mathrm{hd}} / \mathrm{N}_{\mathrm{h}}\right)\right) /\left(\mathrm{G}_{\mathrm{d}} / \mathrm{E}\right)\right)$ where $\mathrm{N}_{\mathrm{h}}$ is the number of differentially expressed genes for which haplotype data was available; $\mathrm{N}_{\text {hd }}$ is the number of differentially expressed genes on different haplotypes; $G_{d}$ Number of genes for which C57BL/6 and A/J have different haplotypes; $\mathrm{E}$ is the Number of Ensembl genes with haplotype data. Values for each variable and condition are shown in Additional File 4 Expression_and_Haplotype.

Identification of pathways in differentially expressed genes Pathways that were overrepresented amongst the differentially expressed genes were identified using KEGG, DAVID and GeneGO and are shown in Additional File 5 GO_KEGG.

\section{Additional material}

Additional file 1 Expression ratios and pplr values for all conditions
for all genes with numbers of upstream SNP and Haplotype block
assignment for A/J.
Additional file 2 Counts of SNP under each probeset and calculations
of their effect on observed expression differences.
Additional file 3 Numbers genes with each number of SNP in their
upstream region and the correlation with expression differences.
Additional file 4 Calculations of number of differentially expressed
genes attributable to haplotype differences.
Additional file 5 GO and KEGG classifications of differentially
expressed genes.
Additional file 6 Description of how allele numbers were assigned to
haplotype blocks.
Additional file 7 Allele assigned to each strain at each haplotype
block.

Abbreviations

eQTL: expression quantative trait locus; KEGG: Kyoto Encyclopaedia of Genes and Genomes.

\section{Authors' contributions}

HN did the SNP analysis and drafted the manuscript; MA managed experimental work on the mice; SA managed microarray work; AA participated in study design and supervised microarray work; $A B$ analysed microarray data and interpreted the analyses; JG designed mouse breeding and supervised mouse work; LH did microarray hybridisations and quality control; $\mathrm{HH}$ analysed microarray data, SJO data interpretation, SJK conceived of the study, interpreted data, redrafted manuscript, supervised study. All authors read and approved the final manuscript.

\section{Acknowledgements}

We thank Leanne Wardlesworth and Leo Zeef of the University of Manchester Core Services unit, Moses Ogugo, John Wambugu of ILRI and the Staff of the ILRI small animal unit for expert technical assistance. This work was supported by The Wellcome Trust [grant number GR066764MA].

\section{Author Details}

1School of Biological Sciences, University of Liverpool, Liverpool, UK, 2Biotechnology Theme, International Livestock Research Institute, Nairobi, Kenya, ${ }^{3}$ National Institute of Animal Science, Rural Development Administration, Suwon, Korea, 4The Roslin Institute and Royal (Dick) School of Veterinary Studies, University of Edinburgh, Roslin, Scotland, UK, ${ }^{5}$ School of Computer Science/Faculty of Life Sciences, University of Manchester, Manchester, UK and ${ }^{6}$ School of Science and Technology, University of New England, Armidale, New South Wales, Australia 
Received: 20 July 2009 Accepted: 7 June 2010

Published: 7 June 2010

\section{References}

1. Yvert G, Brem RB, Whittle J, Akey JM, Foss E, Smith EN, Mackelprang R, Kruglyak L: Trans-acting regulatory variation in Saccharomyces cerevisiae and the role of transcription factors. Nat Genet 2003, 35:57-64.

2. Williams $R$, Chan $E$, Cowley M, Little P: The influence of genetic variation on gene expression. Genome Research 2007, 17:1707-1716.

3. Gibson $G$, Weir B: The quantitative genetics of transcription. Trends Genet 2005, 21:616-623.

4. Breitling R, Li Y, Tesson BM, Fu J, Wu C, Wiltshire T, Gerrits A, Bystrykh LV, de Haan G, Su Al, Jansen RC: Genetical genomics: spotlight on QTL hotspots. PLoS Genet 2008, 4:e1000232.

5. Denver DR, Morris K, Streelman JT, Kim SK, Lynch M, Thomas WK: The transcriptional consequences of mutation and natural selection in Caenorhabditis elegans. Nat Genet 2005, 37:544-548.

6. de Koning D-J, Haley CS: Genetical genomics in humans and model organisms. Trends Genet 2005, 21:377-381.

7. Frazer KA, Eskin E, Kang HM, Bogue MA, Hinds DA, Beilharz EJ, Gupta RV, Montgomery J, Morenzoni MM, Nilsen GB, Pethiyagoda CL, Stuve LL, Johnson FM, Daly MJ, Wade CM, Cox DR: A sequence-based variation map of 8.27 million SNPs in inbred mouse strains. Nature 2007.

8. Iragi F, Clapcott SJ, Kumari P, Haley CS, Kemp SJ, Teale AJ: Fine mapping of trypanosomiasis resistance loci in murine advanced intercross lines. Mamm Genome 2000, 11:645-648.

9. Kemp SJ, Iraqi F, Darvasi A, Soller M, Teale AJ: Localization of genes controlling resistance to trypanosomiasis in mice. Nat Genet 1997 16:194-196.

10. Rathkolb B, Noyes HA, Brass A, Dark P, Fuchs H, Gailus-Durner V, Gibson J, de Angelis MH, Ogugo M, Iraqi F, Kemp SJ, Naessens J, Pope ME, Wolf E, Agaba M: Clinical chemistry of congenic mice with quantitative trait loci for predicted responses to Trypanosoma congolense infection. Infect Immun 2009, 77:3948-3957.

11. Noyes HA, Alimohammadian MH, Agaba M, Brass A, Fuchs H, GailusDurner V, Hulme H, Iraqi F, Kemp S, Rathkolb B, Wolf E, de Angelis MH, Roshandel $D$, Naessens $\mathrm{J}$ : Mechanisms controlling anaemia in Trypanosoma congolense infected mice. PLOS ONE 2009, 4:e5170.

12. Peng $X$, Wood CL, Blalock EM, Chen KC, Landfield PW, Stromberg AJ: Statistical implications of pooling RNA samples for microarray experiments. BMC Bioinf 2003, 4:26.

13. Liu H, Zeeberg BR, Qu G, Koru AG, Ferrucci A, Kahn A, Ryan MC, Nuhanovic A, Munson PJ, Reinhold WC, Kane DW, Weinstein JN: AffyProbeMiner: a web resource for computing or retrieving accurately redefined Affymetrix probe sets. Bioinformatics 2007, 23:2385-2390.

14. Liu X, Milo M, Lawrence ND, Rattray M: A tractable probabilistic model for Affymetrix probe-level analysis across multiple chips. Bioinf 2005, 21:3637-3644.

15. Liu X, Milo M, Lawrence ND, Rattray M: Probe-level measurement error improves accuracy in detecting differential gene expression. Bioinf 2006, 22:2107-2113.

16. Perlegen Mouse SNP browser [http://mouse.cs.ucla.edu/perlegen/]

17. Felsenstein J: PHYLIP (Phylogeny Inference Package) version 3.6. 2005.

18. Behnke JM, Menge DM, Nagda S, Noyes H, Iraqi FA, Kemp SJ, Mugambi RJM, Baker RL, Wakelin D, Gibson JP: Quantitative trait loci for resistance to Heligmosomoides bakeri and associated immunological and pathological traits in mice: comparison of loci on chromosomes 5,8 and 11 in F2 and F6/7 inter-cross lines of mice. Parasitology 2010, 137:311-320.

19. Alberts R, Terpstra P, Li Y, Breitling R, Nap J, Jansen R: Sequence polymorphisms cause many false cis eQTLs. PLoS One 2007, 2:e622.

20. Hovatta I, Zapala MA, Broide RS, Schadt EE, Libiger O, Schork NJ, Lockhart DJ, Barlow C: DNA variation and brain region-specific expression profiles exhibit different relationships between inbred mouse strains: implications for eQTL mapping studies. Genome Biol 2007, 8:R25.

21. Osborne TF, Espenshade PJ: Evolutionary conservation and adaptation in the mechanism that regulates SREBP action: what a long, strange tRIP it's been. Genes Dev 2009, 23:2578-2591.

22. Repa JJ, Turley SD, Lobaccaro JA, Medina J, Li L, Lustig K, Shan B, Heyman RA, Dietschy JM, Mangelsdorf DJ: Regulation of absorption and ABC1- mediated efflux of cholesterol by RXR heterodimers. Science 2000, 289:1524-1529.

23. Ravnskov U: High cholesterol may protect against infections and atherosclerosis. QJM 2003, 96:927-934.

24. Fon Tacer K, Kuzman D, Seliskar M, Pompon D, Rozman D: TNF-alpha interferes with lipid homeostasis and activates acute and proatherogenic processes. Physiol Genomics 2007, 31:216-227.

25. Shimano H: Sterol regulatory element-binding proteins (SREBPs): transcriptional regulators of lipid synthetic genes. Prog Lipid Res 2001, 40:439-452

26. Saito M, Szakall I, Toth R, Kovacs KM, Oros M, Prasad VVTS, Blumenberg M, Vadasz C: Mouse striatal transcriptome analysis: effects of oral selfadministration of alcohol. Alcohol 2004, 32:223-241.

27. Park CC, Ahn S, Bloom JS, Lin A, Wang RT, Wu T, Sekar A, Khan AH, Farr CJ, Lusis AJ, Leahy RM, Lange K, Smith DJ: Fine mapping of regulatory loci for mammalian gene expression using radiation hybrids. Nat Genet 2008, 40:421-429.

28. Lee PD, Ge B, Greenwood CMT, Sinnett D, Fortin Y, Brunet S, Fortin A Takane M, Skamene E, Pastinen T, Hallett M, Hudson TJ, Sladek R: Mapping cis-acting regulatory variation in recombinant congenic strains. Physiol Genomics 2006, 25:294-302.

29. Shao H, Burrage LC, Sinasac DS, Hill AE, Ernest SR, O'Brien W, Courtland HW, Jepsen KJ, Kirby A, Kulbokas EJ, Daly M, Broman K, Lander E, Nadeau $\mathrm{JH}$ : Genetic architecture of complex traits: large phenotypic effects and pervasive epistasis. Proc Natl Acad Sci USA 2008, 105:19910-19914.

30. Fisher P, Hedeler C, Wolstencroft K, Hulme H, Noyes H, Kemp S, Stevens R, Brass A: A systematic strategy for large-scale analysis of genotype phenotype correlations: identification of candidate genes involved in African trypanosomiasis. Nucleic Acids Res 2007, 35:5625-5633.

31. Vladimirov V, Badalova J, Svobodova M, Havelkova H, Hart AA, Blazkova H, Demant P, Lipoldova M: Different genetic control of cutaneous and visceral disease after Leishmania major infection in mice. Infect Immun 2003, 71:2041-2046

32. Churchill GA, Airey DC, Allayee $H$, Angel JM, Attie AD, Beatty J, Beavis WD, Belknap JK, Bennett B, Berrettini W, Bleich A, Bogue M, Broman KW, Buck KJ, Buckler E, Burmeister M, Chesler EJ, Cheverud JM, Clapcote S, Cook MN, Cox RD, Crabbe JC, Crusio WE, Darvasi A, Deschepper CF, Doerge RW, Farber CR, Forejt J, Gaile D, Garlow SJ, Geiger H, Gershenfeld H, Gordon T, Gu J, Gu W, de Haan G, Hayes NL, Heller C, Himmelbauer H, Hitzemann R, Hunter K, Hsu HC, Iraqi FA, Ivandic B, Jacob HJ, Jansen RC, Jepsen KJ, Johnson DK, Johnson TE, Kempermann G, Kendziorski C, Kotb M, Kooy RF, Llamas B, Lammert F, Lassalle JM, Lowenstein PR, Lu L, Lusis A, Manly KF, Marcucio R, Matthews D, Medrano JF, Miller DR, Mittleman G, Mock BA, Mogil JS, Montagutelli X, Morahan G, Morris DG, Mott R, Nadeau JH, Nagase H, Nowakowski RS, O'Hara BF, Osadchuk AV, Page GP, Paigen B, Paigen K, Palmer AA, Pan HJ, Peltonen-Palotie L, Peirce J, Pomp D, Pravenec M, Prows DR, Qi Z, Reeves RH, Roder J, Rosen GD, Schadt EE, Schalkwyk LC, Seltzer Z, Shimomura K, Shou S, Sillanpaa MJ, Siracusa LD, Snoeck HW, Spearow JL, Svenson K, Tarantino LM, Threadgill D, Toth LA, Valdar W, de Villena FP, Warden C, Whatley S, Williams RW, Wiltshire T, Yi N, Zhang D, Zhang M, Zou F: The Collaborative Cross, a community resource for the genetic analysis of complex traits. Nat Genet 2004, 36:1133-1137

33. Reed BD, Charos AE, Szekely AM, Weissman SM, Snyder M: Genome-Wide Occupancy of SREBP1 and Its Partners NFY and SP1 Reveals Novel Functional Roles and Combinatorial Regulation of Distinct Classes of Genes. PLoS Genetics 2008:4.

doi: 10.1186/1471-2164-11-36

Cite this article as: Noyes et al., Genotype and expression analysis of two inbred mouse strains and two derived congenic strains suggest that most gene expression is trans regulated and sensitive to genetic background $B M C$ Genomics 2010, 11:361 